\title{
Marangoni Contraction of Evaporating Sessile Droplets of Binary Mixtures
}

\author{
Stefan Karpitschka, ${ }^{* \dagger}{ }^{\dagger}$ Ferenc Liebig, ${ }^{\ddagger}$ and Hans Riegler $^{\S}$ \\ ${ }^{\dagger}$ Physics of Fluids Group, Faculty of Science and Technology, Mesa+ Institute, University of Twente, 7500 AE Enschede, The \\ Netherlands \\ ${ }^{\ddagger}$ Institute for Chemistry, University of Potsdam, Karl-Liebknecht-Str. 24-25, 14476 Potsdam, Germany \\ ${ }^{\S}$ Max Planck Institute of Colloids and Interfaces, 14424 Potsdam, Germany
}

Supporting Information

ABSTRACT: The Marangoni contraction of sessile drops of a binary mixture of a volatile and a nonvolatile liquid has been investigated experimentally and theoretically. The origin of the contraction is the locally inhomogeneous evaporation rate of sessile drops. This leads to surface tension gradients and thus to a Marangoni flow. Simulations show that the interplay of Marangoni flow, capillary flow, diffusive transport, and evaporative losses can establish a quasistationary drop profile with an apparent nonzero contact angle even if both liquid components individually wet the substrate completely. Experiments with different solvents, initial mass fractions, and gaseous environments reveal a previously unknown universal power-law relation between the apparent contact angle and the relative undersaturation of the ambient atmosphere: $\theta_{\text {app }} \sim\left(\mathrm{RH}_{\mathrm{eq}}-\mathrm{RH}\right)^{1 / 3}$. This experimentally observed power law is in quantitative agreement with simulation results. The exponent can also be inferred from a scaling analysis of the hydrodynamic-evaporative evolution equations of a binary mixture of liquids with different volatilities.

\section{INTRODUCTION}

The evaporation of sessile droplets of mixtures is a ubiquitous natural and industrial process, relevant, e.g., for painting, ${ }^{1}$ for cleaning/drying of semiconductor surfaces, ${ }^{2}$ or for the patterned deposition of solutes on various length scales. ${ }^{3-6}$ Fundamentals of droplet evaporation have been studied over the last decades. ${ }^{7-9}$

Far below the boiling point, the evaporation rate of a liquid is determined by the diffusion of its vapor through the ambient atmosphere. $^{10-13}$ For droplets the evaporation rate is not uniform (Figure 1B) but has a sharp peak at the contact line. ${ }^{14-16}$ This causes for instance the so-called coffee ring effect. ${ }^{14,17,18}$ Besides volumetric flows, evaporation may cause thermal or compositional gradients, both of which may induce Marangoni flows. ${ }^{19-31}$

For binary mixtures, the component with the higher vapor pressure will usually evaporate faster and thus deplete from the contact line region. In general, different liquids have different surface tensions. Thus, nonuniform evaporation causes surface tension gradients and Marangoni flows. ${ }^{30-32}$ Typically, such compositional surface tension gradients are orders of magnitude larger than thermal ones. ${ }^{21}$

If the residual component has a higher surface tension, the Marangoni flow is directed toward the contact line, which may enhance spreading. ${ }^{1,33,34}$ If the residual component has a lower surface tension, the Marangoni flow is directed toward the center of the droplet. If the droplet is placed on a high energy surface that is, in absence of evaporation effects, completely
(A)

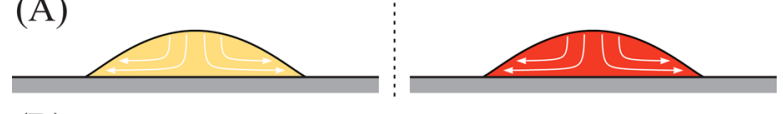

(B)

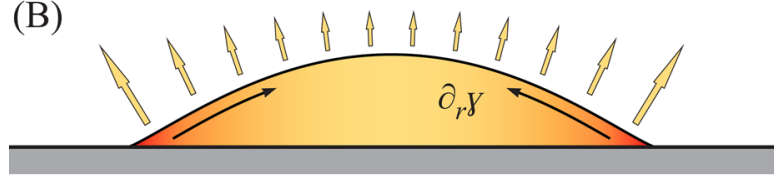

(C)

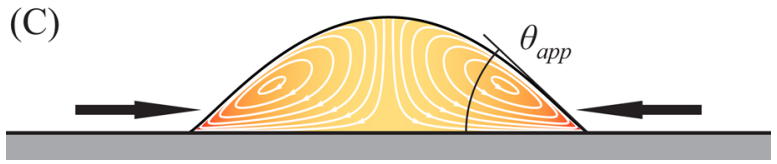

Figure 1. (A) Each sessile drop of a single liquid component spreads completely. (B) A sessile drop with a binary mixture of these components with different surface tensions and volatilities. With evaporation, the nonvolatile component enriches near the contact line. A surface tension gradient develops. (C) The surface tension gradient drives a Marangoni flow. If the volatile component has the higher surface tension, the droplet contracts and exhibits a quasistationary apparent contact angle.

wetted by the droplet, then the contact line is not pinned against a receding motion, and a contraction of the droplet is

Received: March 6, 2017

Revised: April 14, 2017

Published: April 19, 2017 
observed (Figure 1). This "Marangoni drying" is used for instance for cleaning/drying processes in the semiconductor industry. ${ }^{2,36-39}$

Surprisingly, even though the substrate is completely wetted by both components individually (and, in the absence of evaporation, as well by the mixture), droplets of such mixtures may show nonzero apparent contact angles when the highsurface tension component evaporates. ${ }^{30}$ Rather small evaporation rates are sufficient, and the droplets may remain for minutes in such a Marangoni contracted state with a quasistationary apparent contact angle. Eventually, the volatile component will evaporate completely, the contracting flow vanishes, and the droplet will spread. Marangoni contraction has been studied qualitatively in the past. ${ }^{26,30,96,39}$ However, it is still unknown how the apparent contact angle depends on the evaporation properties of the liquid compounds and how it is related to the flow structure in the droplets.

Here we present quantitative experimental investigations on the quasistationary Marangoni contraction of binary mixtures, relate them to a hydrodynamic-evaporative drop model and compare the experimental results to numerical simulations. We present a universal power law relation between the quasistationary contact angle and the relative saturation of the ambient vapor phase, and discuss its origin on the basis of a scaling analysis.

\section{MATERIALS AND METHODS}

The experiments were performed in a temperature-controlled chamber (total volume $\left.\approx 50 \mathrm{~cm}^{3}\right)$ at $(21.0 \pm 0.5){ }^{\circ} \mathrm{C}$. The binary mixtures consisted of a nonvolatile liquid (carbon diols, Sigma, purity >99\%) and a volatile liquid ("Milli-Q" water, resistivity $18 \mathrm{M} \Omega \mathrm{cm}$ ), which mix in all mass fractions $0 \leq \phi \leq 1$. Droplets (initial volume $10 \mu \mathrm{L}$ ) were deposited onto the substrate with a glass syringe (Hamilton GasTight). Water evaporation is rather slow: On a time scale of minutes the drop volume barely changes. Pieces of piranha-cleaned silicon wafers with natural oxide surfaces ( $\mathrm{rms}$ roughness $\approx 0.5 \mathrm{~nm}$ ) served as substrates. ${ }^{40}$ The chamber was continuously flushed with a sufficiently gentle flow of nitrogen gas $(\approx 1 \mathrm{~L} / \mathrm{min}$, purity: 5.0$)$, so that the evaporation rate was dominated by diffusion. The humidity of the nitrogen gas was adjusted by the ratio of mixing dry nitrogen with nitrogen that was saturated with water. The contact angle of the drops was derived from simultaneous imaging side and the top aspects. For more details on the sample preparation, chamber, and the data analysis see refs 40 and 41 .

\section{SPREADING BEHAVIOR AND CONTACT ANGLES}

Figure $2 \mathrm{~A}$ shows the apparent contact angle $\theta_{\text {app }}$ as a function of time after deposition of a droplet of a mixture of butanediol and water at different ambient relative humidities, RH. The pure liquids individually wet the silica surface completely, whereas drops of the mixtures do not (for a certain range of $\mathrm{RH}$ ). Rather, after some initial transient spreading, they reach a quasistationary state with a well-defined $\theta_{\text {app. }}$. This state of quasistationary Marangoni contraction persists for many minutes. During this state $\theta_{\text {app }}$ changes only very slowly as compared to the initial spreading. Due to water evaporation, the diol mass fraction $\phi$ is spatially inhomogeneous and its mean value $\bar{\phi}$, as determined by the total mass of water and diol in the droplet, changes with time. Therefore, we linearly extrapolate $\theta_{\text {app }}$ to $t=0$ (Figure $2 \mathrm{~A}$ ) where $\bar{\phi}$ is precisely known from sample preparation. This $\theta_{\text {app }}^{0}$ decreases with increasing $\mathrm{RH}$ (Figure 2A,B). At a certain equilibrium humidity, $\mathrm{RH}_{\text {eq }}$, the drops spread completely. Measurements show that the spreading in this case follows Tanner's law. ${ }^{42}$ Above $\mathrm{RH}_{\text {eq }}$

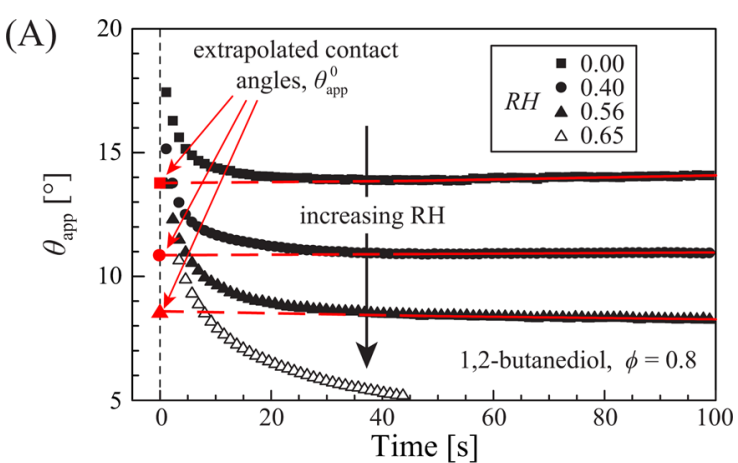

(B)

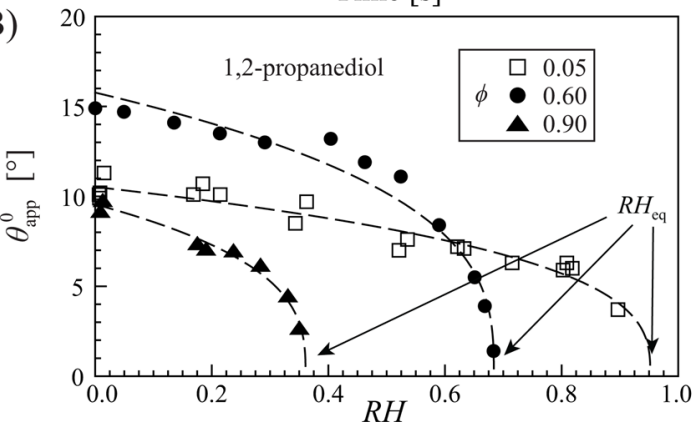

Figure 2. (A) Contact angles as a function of time (1,2-butanediol/ water mixtures). For certain relative humidities, $\mathrm{RH}$, after deposition the drops quickly attain quasistationary apparent contact angles. The linear extrapolation to $t=0$ defines $\theta_{\text {app }}^{0}$ (B) $\theta_{\text {app }}^{0}$ as a function of $\mathrm{RH}$. $\theta_{\text {app }}^{0}=0$ defines the equilibrium relative humidities, $\operatorname{RH}_{\text {eq }}(\bar{\phi})$. The dashed lines are fits according to eq 1. Typical measurement uncertainties are $\pm 1^{\circ}$.

we observed enhanced spreading rates and no stationary apparent contact angle.

Figure $2 \mathrm{~B}$ shows $\theta_{\text {app }}^{0}$ for 1,2-propanediol/water mixtures with mixing ratios $\bar{\phi}=0.05,0.60$, and 0.9 as a function of $R H$. For all $\bar{\phi}$ we observe $\theta_{\text {app }}^{0}$ decreasing with increasing $\mathrm{RH}$. Intermediate $\bar{\phi}$ lead to the largest $\theta_{\text {app }}^{0}$. $\mathrm{RH}_{\mathrm{eq}}$ decreases with increasing $\phi$. Experiments were also performed for aqueous mixtures of ethylene glycol, 1,2-propanediol, and 1,2butanediol, with diol mass fractions ranging from $0.5 \%$ to $90 \%$. The results are documented in the Supporting Information $^{35}$ and follow the same trends as the ones depicted in Figure 2A,B.

The dashed lines in Figure $2 \mathrm{~B}$ are least-squares fits to the data with

$$
\theta_{\text {app }}^{0}(\bar{\phi})=A(\bar{\phi})\left(\mathrm{RH}_{\mathrm{eq}}(\bar{\phi})-\mathrm{RH}\right)^{1 / 3}
$$

The exponent of $1 / 3$ is motivated by simulation results (see below; experimental data would allow for exponents between 0.25 and 0.4). The fit parameters are $A(\bar{\phi})$ and $\mathrm{RH}_{\mathrm{eq}}(\bar{\phi}) . A(\bar{\phi})$ is a prefactor that accounts for $\bar{\phi}$ and for other substancespecific properties (surface tension $\gamma(\bar{\phi})$, etc.). $\mathrm{RH}_{\mathrm{eq}}(\bar{\phi})$ denotes the relative humidity, at which the drops just spread completely. $\mathrm{RH}$ is the (measured) relative humidity inside the chamber, where for contraction, $\mathrm{RH}<\mathrm{RH}_{\mathrm{eq}}(\bar{\phi})$. In diffusion limited conditions, $\mathrm{RH}_{\mathrm{eq}}(\bar{\phi})-\mathrm{RH}$ is linearly related to the total evaporation rate. ${ }^{43,44}$

Figure 3A shows $A(\bar{\phi})$ as a function of the initial diol mass fraction $\bar{\phi}$ for three different diols. $A(\bar{\phi})$ is derived from the experimental data assuming a behavior according to eq 1 . In the range of $0.3 \lesssim \bar{\phi} \lesssim 0.8, A(\bar{\phi})$ changes only very little. With $\bar{\phi}$ approaching 0 or $1, A(\bar{\phi})$ decreases (contraction is not observed for single-component droplets). While the diol 

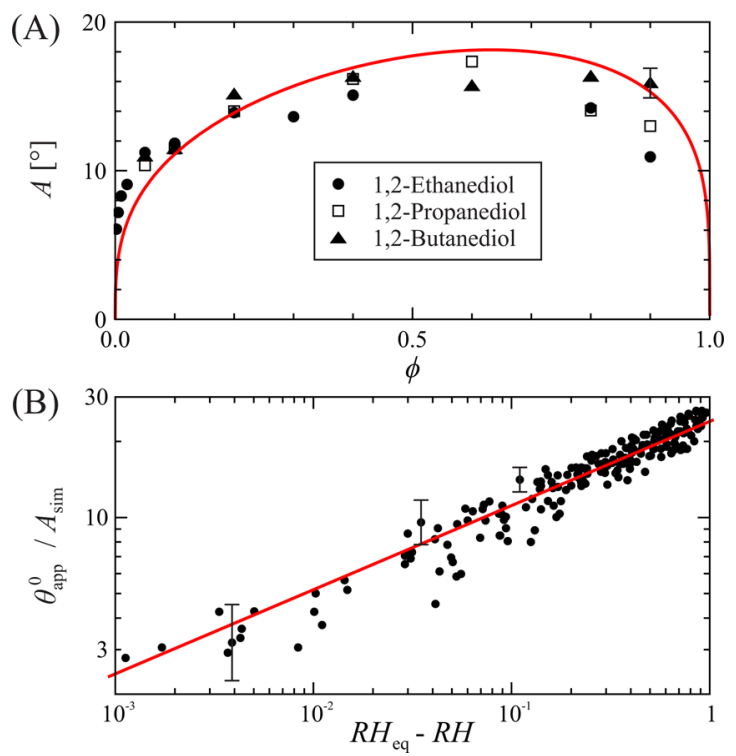

Figure 3. (A) Prefactor $A(\bar{\phi})$ as a function of the diol mass fraction, $\bar{\phi}$, for three different diol/water mixtures. $A(\bar{\phi})$ is extracted from experimental data according to eq 1 . The red line assumes ideal behavior according to eq 7. (B) $\theta_{\text {app }}^{0}(\bar{\phi})$ as a function of $\mathrm{RH}_{\text {eq }}(\bar{\phi})-$ $\mathrm{RH}$. The experimentally derived $\theta_{\text {app }}^{0}(\bar{\phi})$ is scaled by $A(\bar{\phi})$ from simulations (red line in A). The red line shows the $1 / 3$ power law resulting from the simulations.

species has a comparatively small influence on $A(\bar{\phi})$, there is a noticeable trend of lower surface tension diols having somewhat larger prefactors (1,2-butandiol, $\gamma=36 \mathrm{mN} / \mathrm{m}$, vs 1,2-ethandiol, $\gamma=46 \mathrm{mN} / \mathrm{m})$. This supports the reasoning that the contraction is actually caused by Marangoni flows. The red line indicates a simulation result (see below). Figure 3B) shows the measured $\theta_{\text {app }}$ as scaled to the simulated $A(\bar{\phi})$ as a function of $\mathrm{RH}_{\mathrm{eq}}-\mathrm{RH}$. Again, the red line represents the simulation result, $\theta_{\text {app }} \sim\left(\mathrm{RH}_{\mathrm{eq}}-\mathrm{RH}\right)^{1 / 3}$.

\section{SIMULATIONS}

In lubrication approximation, ${ }^{45,46}$ the evolution equations for height $h(r)$ and vertically averaged mass fraction $\phi(r)$ of a radially symmetric thin liquid film are

$$
\begin{aligned}
& \partial_{\mathrm{t}} h=-\frac{1}{r} \partial_{\mathrm{r}}\left\{r\left[\Phi_{\mathrm{c}}+\Phi_{\mathrm{m}}+J /(2 \pi r)\right]\right\} \\
& \partial_{\mathrm{t}}(\phi h)=-\frac{1}{r} \partial_{\mathrm{r}}\left\{r\left[\phi \Phi_{\mathrm{c}}+\phi \Phi_{\mathrm{m}}+\Phi_{\mathrm{d}}\right]\right\}
\end{aligned}
$$

The capillary, Marangoni, and diffusive fluxes are

$$
\Phi_{\mathrm{c}}=-\frac{h^{3}}{3 \eta} \partial_{\mathrm{r}} p, \Phi_{\mathrm{m}}=\gamma^{\prime}(\phi) \frac{h^{2}}{2 \eta} \partial_{\mathrm{r}} \phi, \Phi_{\mathrm{d}}=-D h \partial_{\mathrm{r}} \phi
$$

$\eta$ is the liquid viscosity and $D$ is the diffusivity of the two components in the liquid. $\gamma(\phi)$ is the composition-dependent surface tension and the prime denotes differentiation with respect to $\phi$. The pressure, $p$, contains contributions from capillarity and from surface forces:

$$
p=-\gamma(\phi)\left\{\frac{1}{r} \partial_{\mathrm{r}}\left(r \partial_{\mathrm{r}} h\right)+\frac{a^{2}}{h^{3}}\right\}
$$

$a$ is the van-der-Waals length which determines the magnitude of the surface forces. The surface force term generates a complete wetting scenario that is independent of the liquid type and/or composition. For simplicity we assume a linear $\gamma(\phi)$ and chose $\gamma^{\prime}(\phi)=-20 \mathrm{mN} / \mathrm{m}$ to reflect the average behavior of the experiments in the range $0.1 \lesssim \phi \lesssim 0.3$. The diols are small molecules with negligible surface activity, ${ }^{40}$ and we may use a direct relation between surface tension and bulk concentration. Due to the presence of strong solutal Marangoni flows, the influence of thermal gradients is orders of magniude smaller and will be ignored.

We assume a negligible vapor pressure of the diols, and $J$ in eq 2 is the total water evaporation rate from the center up to radius $r$. We calculate $J$ after the quasistationary, nonlocal scheme for diffusion limited evaporation from ref 46 by an integral transformation of the local vapor $p_{\mathrm{v}}$ :

$$
J=-\frac{D_{\mathrm{v}} M_{\mathrm{w}}}{\rho R_{\mathrm{g}} T} \int_{0}^{\infty} \kappa\left(r, r^{\prime}\right) \partial_{\mathrm{r}^{\prime}}\left[p_{\mathrm{v}}(\phi)+\frac{p_{\mathrm{v}}(\phi) p M_{\mathrm{w}}}{\rho R_{\mathrm{g}} T}\right] \mathrm{d} r^{\prime}
$$

with the kernel $\kappa\left(r, r^{\prime}\right)$ (a combination of elliptic integrals, see the Supporting Information for details ${ }^{35}$ ), the vapor diffusivity $D_{\mathrm{v}}$, the gas constant $R_{\mathrm{g}}$, and the temperature $T$. In addition to ref 46, we also take the compositional dependence of the saturation vapor pressure into account. Assuming a thermodynamically ideal mixture behavior, the partial vapor pressure of water, $p_{\mathrm{v}}(\phi)$, in equilibrium with a diol/water mixture is ${ }^{47}$

$$
p_{\mathrm{v}}(\phi)=p_{\mathrm{sat}} \frac{M_{\mathrm{D}}(1-\phi)}{M_{\mathrm{w}}+\left(M_{\mathrm{D}}-M_{\mathrm{w}}\right)(1-\phi)}
$$

with $p_{\text {sat }}$ as partial vapor pressure of pure water. $M_{\mathrm{w}}$ and $M_{\mathrm{D}}$ are the molecular weights of water and diol, respectively. $\mathrm{RH}_{\mathrm{eq}}=$ $p_{\mathrm{v}} / p_{\text {sat }}$, calculated with eq 7 , shows a monotonic decrease of $\mathrm{RH}_{\mathrm{eq}}$ with $\phi$ and agrees quite well with the experimental $\mathrm{RH}_{\mathrm{eq}}$ (see the Supporting Information for experimental data ${ }^{35}$ ). Simulations were initialized with droplets of homogeneous composition and various initial average mass fractions $\phi \in$ $\left[10^{-3} \ldots 0.9\right]$. The droplets were placed as spherical caps with an initial apparent contact angle of $20^{\circ}$ on top of a precursor film that was 5 orders of magnitude thinner than the bulk droplet height. The pressure-dependent term in eq 6, together with the disjoining pressure from eq 5 ensures equilibrium between precursor film and vapor. This establishes an effective "no-flux" boundary condition for the vapor at the substrate around the droplet. Simulations were run until complete evaporation; thus, we obtain droplets with similar mean compositions but different age and volume. For further details on initial and boundary conditions, please refer to the Supporting Information. ${ }^{35}$

Figure 4 presents simulation data for quasistationary droplets with $\bar{\phi} \approx 0.15(\mathrm{~A}, \mathrm{~B})$ and $\bar{\phi} \approx 0.85(\mathrm{C}, \mathrm{D})$ in a dry environment (further plots for other average mass fractions can be found in the supplement; ${ }^{35}$ they show the same characteristic features as those shown in Figure 4). Here, $\bar{\phi}$ denotes the average mass fraction of the droplet:

$$
\bar{\phi}=\frac{\int_{0}^{R} \phi h r \mathrm{~d} r}{\int_{0}^{R} h r \mathrm{~d} r}
$$

Water evaporation (A, C, solid) locally enriches the diol (B, D, color code). Diol enrichment causes (I) an inward Marangoni flow that contracts the drop and (II) a reduced evaporation rate $j$ (A, C solid) near the contact line (as compared to the 

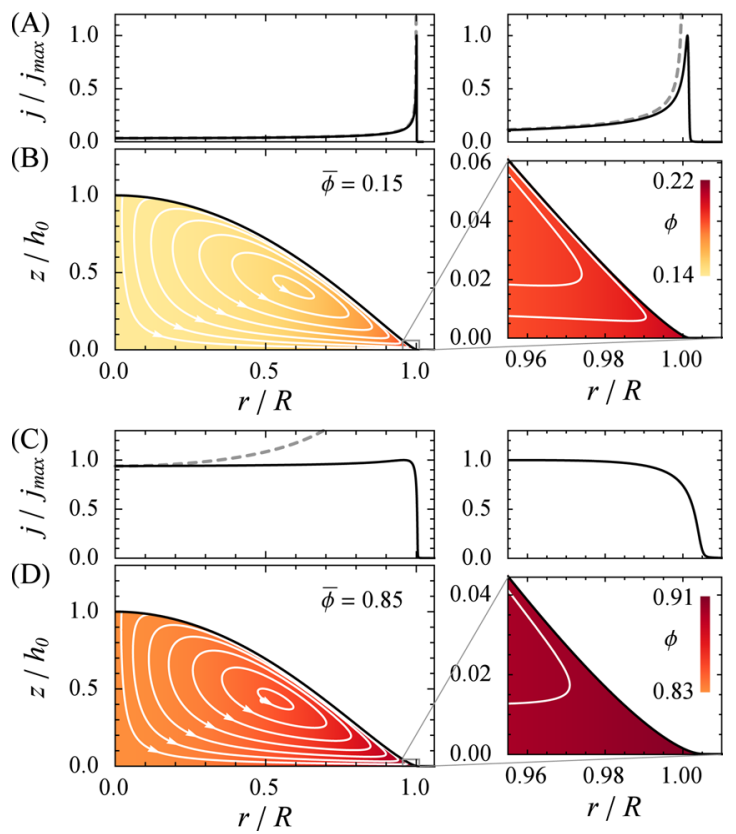

Figure 4. Simulation snapshots of droplets in quasistationary conditions for small and large average diol mass fraction (A,B: $\bar{\phi}=$ 0.15 ; C,D: $\bar{\phi}=0.85$ ). The ambient humidity is $R H=0$ in both cases. (A and C) show the local evaporation rate as a function of $r$ (solid) compared to the contiuum theory $j \sim\left(R^{2}-r^{2}\right)^{-1 / 2}$ for onecomponent droplets ${ }^{14}$ (dashed). (B and D) show drop profiles (black), stream lines (white), and local mass fractions $\phi$ (color code). The panels on the right show zooms to the contact line region. The color scale of the local $\phi$ is given on the right panels. The minimum local $\phi$ is found at $r=0$ and remains close to $\phi$. The maximum $\phi$ is obvserved near the contact line. For small $\bar{\phi}$, evaporation follows closely the ideal one-component solution, while at high $\bar{\phi}$, diol enrichment leads to an almost homogeneous evaporation rate.

continuum theory (dashed) for one-component droplets ${ }^{14}$ ). For small $\bar{\phi}$, the droplet profile $h(r)$ (B, black) is close to a spherical cap. Deviations occur only close to the contact line but expand with increasing $\bar{\phi}(\mathrm{D})$.

Most remarkable, the simulations reveal quasistationary apparent contact angles, for which the height-integrated capillary and Marangoni fluxes balance each other in a convection roll ( $\mathrm{B}, \mathrm{D}$, white stream lines). Only on a very slow time scale, droplet volume and average composition change due to evaporative losses. In agreement with the experimental data, $\theta_{\text {app }}$ is size independent and follows the 1/3power law of eq 1 (Figure 3B, red line).

At small $\bar{\phi}$ (Figure $4 \mathrm{~B}$ ), the enrichment is limited by the small amount of available diol. At large $\bar{\phi}$, the diol enrichment near the contact line leads to an almost homogeneous evaporation rate without a sharp peak at the contact line (Figure $4 \mathrm{C}$ ): enrichment is self-limiting due to its impact on the evaporation rate as can be seen from the dependence of $p_{\mathrm{v}}$ on $\phi$ (eq 7 and Figure S2 in the Supporting Information ${ }^{35}$ ). $A(\bar{\phi})$ (Figure $3 \mathrm{~A}$, red line) is largest for intermediate $\bar{\phi}$. At small $\bar{\phi}$, the simulated $A(\bar{\phi})$ is smaller than the measured one, whereas for intermediate $\bar{\phi}$, it is somewhat larger. This correlates well with the experimentally observed surface activity of the diol, with $\underline{\gamma^{\prime}}(\bar{\phi})<-20 \mathrm{mN} / \mathrm{m}$ for small $\bar{\phi}$ and $\gamma^{\prime}(\bar{\phi})>$ $-20 \mathrm{mN} / \mathrm{m}$ for $\bar{\phi}$ close to $1 .^{40}$

Figure $3 \mathrm{~B}$ shows the experimental data $\theta_{\mathrm{app}}^{0}(\bar{\phi})$ scaled to the simulated prefactor $A_{\text {sim }}$ as a function of $\mathrm{RH}_{\mathrm{eq}}-\mathrm{RH}\left(\mathrm{RH}_{\mathrm{eq}}\right.$ is obtained from the fit of eq 1 ). The red line corresponds to the
$1 / 3$ power law found in the simulations. For $\mathrm{RH} \rightarrow \mathrm{RH}_{\mathrm{eq}} \phi(r)$ becomes increasingly homogeneous, which reduces the contractile Marangoni flow and quasistationarity is observed for smaller $\theta_{\text {app }}$.

\section{DISCUSSION}

Why does the apparent contact angle of Marangoni-contracted droplets scale according to eq 1 ? This can be inferred from the hydrodynamic-evaporative behavior described by eqs $2-5$. The total evaporation rate $J$ is small compared to the hydrodynamic fluxes. Evaporative enrichment and diffusive dilution approximately balance each other. At the same time, capillary and Marangoni fluxes also compensate each other. This leads to a quasistationary, approximately spherical cap shape of the droplet with a quasistationary composition gradient.

For quasistationarity, the comparison of eqs 2 and 3 yields the balance between enrichment and diffusive dilution:

$$
\Phi_{\mathrm{d}} \sim \phi J / r
$$

From eq 4 follows $\Phi_{\mathrm{m}}=\frac{\gamma^{\prime}(\phi)}{2 \eta D} \Phi_{\mathrm{d}}$. Thus, the scaling behavior of $\Phi_{\mathrm{m}}$ in eq 2 can be expressed in terms of $J$. Quasistationarity once more implies that the time derivative on the left-hand side of eq 2 becomes vanishingly small, and that the total evaporation $J$ is much smaller than each of the hydrodynamic fluxes $\Phi_{c}$ and $\Phi_{m}$. Consequently, these fluxes approximately balance each other, which leads to

$$
\Phi_{c} \sim \frac{\gamma^{\prime}(\phi)}{2 \eta D} \frac{\phi h J}{r}
$$

On the other hand $\Phi_{c} \sim h_{0}{ }^{4} / R^{3}$ (eqs 4 and 5), where we used droplet height $h_{0}$ and radius $R$ as the characteristic vertical and horizontal scales, respectively. This, together with $J \sim R\left(\mathrm{RH}_{\mathrm{eq}}\right.$ $-\mathrm{RH}){ }^{46}$ results in

$$
h_{0}{ }^{3} / R^{3} \sim\left(\mathrm{RH}_{\mathrm{eq}}-\mathrm{RH}\right)
$$

The apparent contact angle $\theta_{\text {app }} \approx 2 h_{0} / R$ is a measure for the droplet aspect ratio, ergo

$$
\theta_{\text {app }} \sim\left(\mathrm{RH}_{\mathrm{eq}}-\mathrm{RH}\right)^{1 / 3}
$$

This result is different from existing literature ${ }^{30}$ describing a linear relation between $\cos \theta_{\text {app }}$ and RH, i.e., $\theta_{\text {app }} \sim(1-$ $\mathrm{RH})^{1 / 2}$.

We note here that the present case is quite different from the typical three stage evaporation behavior of droplets on partially wetted substrates with contact angle hysteresis ${ }^{48}$ or the spreading behavior of aqueous superspreading surfactant solutions. ${ }^{49}$ In the present case, there is no pinned stage at all, and the droplets are highly mobile. The apparent contact angle is increased by a Marangoni flow, in the absence of which the droplets would fully wet the substrate.

\section{CONCLUSIONS}

Sessile drops of binary liquid mixtures of a volatile and a nonvolatile component of different surface tensions can assume a Marangoni contracted state with a quasistationary, nonzero apparent contact angle, $\theta_{\text {app}}$, even if both liquids individually wet the substrate completely. We present experimental data on how this $\theta_{\text {app }}$ depends on the mass fraction of the mixture and the ambient partial pressure of the volatile component. The surface tension gradient results from the locally nonuniform evaporation rate of sessile droplets. We find a universal power 
law that links $\theta_{\text {app }}$ to the relative under-saturation of the ambient vapor with an exponent of $1 / 3$.

The contracted drop state with the nonzero apparent contact angle is explained by a quasistationary balance between capillary and Marangoni fluxes, corroborated by simulations that quantitatively match experimental observations. It is not caused by the effective substrate surface tension around the droplet. ${ }^{30}$ It is also very different from the contact angle modification due to fast evaporation in the case of pure liquids. ${ }^{46}$

The characteristic exponent of $1 / 3$, found by experiment and simulation, was traced back to the scaling behavior of the evolution equations for evaporating sessile drops.

\section{ASSOCIATED CONTENT}

\section{S Supporting Information}

The Supporting Information is available free of charge on the ACS Publications website at DOI: 10.1021/acs.langmuir.7b00740.

Document containing details on experimental and numerical methods. (PDF)

\section{AUTHOR INFORMATION}

\section{Corresponding Author}

*E-mail: karpitschka@googlemail.com.

\section{ORCID}

Stefan Karpitschka: 0000-0001-9210-2589

\section{Notes}

The authors declare no competing financial interest.

\section{ACKNOWLEDGMENTS}

We acknowledge helpful discussions with Jacco Snoeijer, Uwe Thiele, and Len Pismen. We also thank H. Möhwald for scientific advice and general support. S.K. acknowledges the hospitality of the Isaac Newton Institute for Mathematical Sciences at the University of Cambridge during the research program "Mathematical Modeling and Analysis of Complex Fluids and Active Media in Evolving Domains" (2013). S.K. and F.L. were supported by LAM Research AG, Austria.

\section{REFERENCES}

(1) Wilson, S. The levelling of paint films. IMA J. Appl. Math. 1993, 50, 149-166.

(2) Leenaars, A.; Huethorst, J.; van Oekel, J. Marangoni drying: a new extremely clean drying process. Langmuir 1990, 6, 1701-1703.

(3) Yella, A.; Tahir, M.; Meuer, S.; Zentel, R.; Berger, R.; Panthöfer, M.; Tremel, W. Synthesis, Characterization, and Hierarchical Organization of Tungsten Oxide Nanorods: Spreading Driven by Marangoni Flow. J. Am. Chem. Soc. 2009, 131, 17566-17575.

(4) Harris, D.; Lewis, J. Marangoni effects on evaporative lithographic patterning of colloidal films. Langmuir 2008, 24, 3681-3685.

(5) Wang, Y.; Song, Y.; Watanabe, S.; Zhang, S.; Li, D.; Zhang, X. Stitching Chemically Converted Graphene on Solid Surfaces by Solvent Evaporation. ACS Appl. Mater. Interfaces 2012, 4, 6443-6339.

(6) Park, J.; Moon, J. Control of Colloidal Particle Deposit Patterns within Picoliter Droplets Ejected by Ink-Jet Printing. Langmuir 2006, $22,3506-3513$

(7) Shahidzadeh-Bonn, N.; Rafai, A.; Azouni, A.; Bonn, D. Evaporating droplets. J. Fluid Mech. 2006, 549, 307-313.

(8) Semenov, S.; Starov, V.; Velarde, M.; Rubio, R. Droplets evaporation: Problems and solutions. Eur. Phys. J.: Spec. Top. 2011, 197, 265-278.

(9) Gelderblom, H.; Stone, H.; Snoeijer, J. Stokes flow in a drop evaporating from a liquid subphase. Phys. Fluids 2013, 25, 102102.
(10) Maxwell, J. In The scientific papers of James Clerk Maxwell; Niven, W. D., Ed.; Cambridge University Press: Cambridge, U.K., 1890; Vol. 2; pp 625-646.

(11) Langmuir, I. The evaporation of small spheres. Phys. Rev. 1918, 12, 368-370.

(12) Picknett, R.; Bexon, R. The evaporation of sessile or pendant drops in still air. J. Colloid Interface Sci. 1977, 61, 336-350.

(13) Sefiane, K.; Wilson, S.; David, S.; Dunn, G.; Duffy, B. On the effect of the atmosphere on the evaporation of sessile droplets of water. Phys. Fluids 2009, 21, 062101.

(14) Deegan, R.; Bakajin, O.; Dupont, T.; Huber, G.; Nagel, S.; Witten, T. Capillary flow as the cause of ring stains from dried liquid drops. Nature 1997, 389, 827-829.

(15) Deegan, R. Pattern formation in drying drops. Phys. Rev. E: Stat. Phys., Plasmas, Fluids, Relat. Interdiscip. Top. 2000, 61, 475-485.

(16) Gelderblom, H.; Bloemen, O.; Snoeijer, J. Stokes flow near the contact line of an evaporating drop. J. Fluid Mech. 2012, 709, 69-84.

(17) Marin, A.; Gelderblom, H.; Lohse, D.; Snoeijer, J. Order-todisorder transition in ring-shaped colloidal stains. Phys. Rev. Lett. 2011, 107, 085502.

(18) Yunker, P.; Still, T.; Lohr, M.; Yodh, A. G. Suppression of the coffee-ring effect by shape-dependent capillary interactions. Nature 2011, 476, 308-311.

(19) Scriven, L.; Sternling, C. The Marangoni Effects. Nature 1960, $187,186-188$.

(20) Hu, H.; Larson, R. Marangoni effect reverses coffee-ring depositions. J. Phys. Chem. B 2006, 110, 7090-7094.

(21) Hu, H.; Larson, R. G. Analysis of the effects of marangoni stresses on the microflow in an evaporating sessile droplet. Langmuir 2005, 21, 3972-3980.

(22) MacDonald, B.; Ward, C. Onset of Marangoni convection for evaporating sessile droplets. J. Colloid Interface Sci. 2012, 383, 198207.

(23) Karapetsas, G.; Matar, O.; Valluri, P.; Sefiane, K. Convective rolls and hydrothermal waves in evaporating sessile drops. Langmuir 2012, 28, 11433-11439.

(24) Girard, F.; Antoni, M.; Sefiane, K. On the Effect of Marangoni Flow on Evaporation Rates of Heated Water Drops. Langmuir 2008, 24, 9207-9210.

(25) Ristenpart, W.; Kim, P.; Domingues, C.; Wan, J.; Stone, H. Influence of substrate conductivity on circulation reversal in evaporating drops. Phys. Rev. Lett. 2007, 99, 234502.

(26) Majumder, M.; Rendall, C.; Eukel, J.; Wang, J.; Behabtu, N.; Pint, C.; Liu, T.-Y.; Orbaek, A.; Mirri, F.; Nam, J.; Barron, A.; Hauge, R.; Schmidt, H.; Pasquali, M. Overcoming the coffee-stain effect by compositional marangoni-flow assisted drop-drying. J. Phys. Chem. B 2012, 116, 6536-6542.

(27) Christy, J.; Hamamoto, Y.; Sefiane, K. Flwo transition within an evaporating binary mixture sessile drop. Phys. Rev. Lett. 2011, 106, 205701.

(28) Still, T.; Yunker, P.; Yodh, A. Surfactant-Induced Marangoni Eddies Alter the Coffee-Rings of Evaporating Colloidal Drops. Langmuir 2012, 28, 4984-4988.

(29) Marin, A.; Liepelt, R.; Rossi, M.; Kähler, C. J. Surfactant-driven flow transitions in evaporating droplets. Soft Matter 2016, 12, 15931600.

(30) Cira, N.; Benusiglio, A.; Prakash, M. Vapour-mediated sensing and motility in two-component droplets. Nature 2015, 519, 446-450.

(31) Kim, H.; Boulogne, F.; Um, E.; Jacobi, I.; Button, E.; Stone, H. Controlled Uniform Coating from the Interplay of Marangoni Flows and Surface-Adsorbed Macromolecules. Phys. Rev. Lett. 2016, 116, 124501 .

(32) Soulie, V.; Karpitschka, S.; Lequien, F.; Prené, P.; Zemb, T.; Möhwald, H.; Riegler, H. The evaporation behavior of sessile droplets from aqueous saline solutions. Phys. Chem. Chem. Phys. 2015, 17, 22296.

(33) Cachile, M.; Benichou, O.; Cazabat, A. Evaporating Drops of completely wetting liquids. Langmuir 2002, 18, 7985-7990. 
(34) Gotkis, Y.; Ivanov, I.; Murisic, N.; Kondic, L. Dynamic structure formation at the fronts of volatile liquid drops. Phys. Rev. Lett. 2006, $97,186101$.

(35) The Supporting Information is available free of charge on the ACS Publications website at DOI: 10.1021/acs.langmuir.7b00740.

(36) Marra, J.; Huethorst, J. Physical principles of Marangoni drying. Langmuir 1991, 7, 2748-2755.

(37) Matar, O.; Craster, R. Models for Marangoni drying. Phys. Fluids 2001, 13, 1869-1883.

(38) Thess, A.; Boos, W. A model for Marangoni drying. Phys. Fluids 1999, 11, 3852-3855.

(39) Huethorst, J.; Marra, J. Motion of marangoni-contracted water drops across inclined hydrophilic surfaces. Langmuir 1991, 7, 27482755.

(40) Karpitschka, S.; Riegler, H. Quantitative Experimental Study on the Transition between Fast and Delayed Coalescence of Sessile Droplets with Different but Completely Miscible Liquids. Langmuir 2010, 26, 11823-11829.

(41) Karpitschka, S.; Riegler, H. Non-coalescence of sessile drops from different but miscible liquids: Hydrodynamic analysis of the twin drop contour as self stabilizing, traveling wave. Phys. Rev. Lett. 2012, 109, 066103.

(42) Tanner, L. The spreading of silicone oil drops on horizontal surfaces. J. Phys. D: Appl. Phys. 1979, 12, 1473-1484.

(43) Stauber, J.; Wilson, S.; Duffy, B.; Sefiane, K. On the lifetime of evaporating droplets. J. Fluid Mech. 2014, 744, R2.

(44) Popov, Y. O. Evaporative deposition patterns: Spatial dimensions of the deposit. Phys. Rev. E 2005, 71, 036313.

(45) Oron, A.; Davis, S.; Bankoff, S. Long-scale evolution of thin liquid films. Rev. Mod. Phys. 1997, 69, 931-980.

(46) Eggers, J.; Pismen, L. Nonlocal description of evaporating drops. Phys. Fluids 2010, 22, 112101.

(47) Atkins, P.; de Paula, J. Physical Chemistry; Oxford University Press: New York, 2009.

(48) Semenov, S.; Starov, V.; Rubio, R.; Velarde, M. Evaporation of sessile water droplets: Universal behavior in presence of contact angle hysteresis. Colloids Surf., A 2011, 392, 135-144.

(49) Semenov, S.; Trybalba, A.; Agogo, H.; Kovalchuk, N.; Ortega, F.; Rubio, R.; Starov, V.; Velarde, M. Evaporation of Droplets of Surfactant Solutions. Langmuir 2013, 29, 10028. 